\title{
Assessing right ventricular function in patients with pulmonary hypertension based on noninvasive measurements: correlation between cardiac MRI, ultrasonic cardiogram, multidetector $\mathrm{CT}$ and right heart catheterization
}

\author{
Xiaojuan Guo ${ }^{1 *}$, Min Liu', Zhanhong Ma', tao jiang ${ }^{1}$, Yuanhua Yang ${ }^{2}$, zhenguo zhai ${ }^{2}$, Renyou zhai ${ }^{1}$, \\ Tianjing Zhang ${ }^{3}$
}

From 18th Annual SCMR Scientific Sessions

Nice, France. 4-7 February 2015

\section{Background}

The assessment of right ventricular function is of great importance in the management of patients with pulmonary hypertension $(\mathrm{PH})$. Our aim is to compare the value of cardiac MRI (CMRI), ultrasonic cardiogram (CUS), multidetector CT (MDCT) in assessing right ventricular function, and to evaluate the correlation between parameters derived by MRI, CUS, MDCT and the indicators of right ventricular function derived by right heart catheterization (RHC) in patients with $\mathrm{PH}$.

\section{Methods}

Thirty one consecutive patients with $\mathrm{PH}$ (17 males and 14 females; mean age, $54.61 \pm 11.74$ years; range, 33 to 76 years) were prospectively enrolled.All patients underwent $\mathrm{RCH}$ to get hemodynamic parameters .Then, calculations including pulmonary vascular resistance index (PVRI), right cardiac work index (RCWI), right ventricular stroke work index (RVSWI) were performed. All patients underwent CMRI to get parameters including right ventricular end-systolic volume (ESV), end-diastolic dimension (EDV), stroke volume (SV), ejection fraction (EF), the cardiac muscle mass (CMS). 28 patients underwent CUS to get these parameters including right ventricular Tei index, right ventricular fractional area change (RV FAC), RV-ESV, RV-EDV. And 25 patients

${ }^{1}$ Department of Radiology, Beijing Chao-Yang Hospital, Capital Medical University, Beijing, China

Full list of author information is available at the end of the article underwent MDCT to get these parameters including right /left ventricular internal diameter $(\mathrm{RVd} / \mathrm{LVd})$, right /left ventricular diastole maximum area (RVa/LVa), cobb angle, right ventricular free wall thickness (RVWT). All examinations were executed within 7days. These parameters obtained by MRI, CUS and MDCT were correlated with those of $\mathrm{RCH}$ respectively by Spearman or Pearson correlation analysis.

\section{Results}

Most parameters of RV function derived by CMRI, CUS, MDCT correlated moderately with mPAP, PVRI. mPAP had strong correlation with MRI-ventricular mass index $(\mathrm{VMI})(r=0.528, P=0.002)$, RVWT $(r=0.554, P=0.005)$; PVRI had strong correlation with MRI-EF $(r=-0.647$, $P=0.000), \quad$ MRI-VMI $(r=-0.567, P=0.001), \quad$ CT $-\mathrm{RVd}$ $(r=0.536, P=0.006), \mathrm{RVd} / \mathrm{LVd}(r=0.530, P=0.006)$, CTCobb angle $(r=0.608, \quad P=0.001), \quad \operatorname{RVWT}(r=0.586$, $P=0.003)$. Table 1 showed the correlation of right ventricular function between CMRI, CUS, MDCT and RHC. RHC-SV had the strong correlation with MRI-EF $(r=0.557, P=0.001)$, CT-RVd $(r=-0.502, P=0.011)$, CTCobb angle $(r=-0.503, P=0.003)$.

\section{Conclusions}

The parameters obtained by MRI, CUS and MDCT had moderate correlation with RHC-SV. MRI-EF was the best parameter to reflect RV function which could be used to exactly evaluate RV function in patient with $\mathrm{PH}$. 
Table 1 Correlation coefficients of right ventricular function parameters between CMRI, CUS,M DCT and RHC.

\begin{tabular}{|c|c|c|c|c|c|c|c|c|}
\hline & $6 \mathrm{MWD}$ & & RHC-SV & & RCWI & & RVSWI & \\
\hline & $r$ & $P$ & $r$ & $P$ & $r$ & $P$ & $r$ & $\mathrm{P}$ \\
\hline MRI-SV(n=31) & 0.073 & 0.713 & 0.457 & 0.010 & 0.322 & 0.077 & 0.324 & 0.075 \\
\hline MRI-EF & 0.473 & 0.011 & 0.557 & 0.001 & 0.389 & 0.031 & 0.307 & 0.093 \\
\hline MRI-VMI & -0.472 & 0.011 & -0.444 & 0.012 & -0.259 & 0.159 & -0.173 & 0.351 \\
\hline US-SV(n=28) & -0.268 & 0.186 & 0.016 & 0.937 & -0.201 & 0.305 & -0.071 & 0.718 \\
\hline US-EF & -0.019 & 0.923 & 0.406 & 0.026 & 0.015 & 0.939 & 0.023 & 0.905 \\
\hline US-Tei & -0.175 & 0.448 & -0.480 & 0.024 & -0.387 & 0.075 & -0.419 & 0.042 \\
\hline US-RVFAC & 0.046 & 0.822 & 0.385 & 0.043 & 0.289 & 0.136 & 0.199 & 0.311 \\
\hline$C T-R V d(n=25)$ & -0.373 & 0.079 & -0.502 & 0.011 & -0.486 & 0.014 & -0.468 & 0.018 \\
\hline CT-RVa & -0.351 & 0.109 & -0.488 & 0.016 & -0.342 & 0.102 & -0.362 & 0.082 \\
\hline CT-Cobb angle & -0.569 & 0.004 & -0.503 & 0.003 & -0.430 & 0.025 & -0.395 & 0.041 \\
\hline CT-RWWT & -0.098 & 0.666 & -0.279 & 0.186 & -0.024 & 0.911 & 0.023 & 0.914 \\
\hline
\end{tabular}

6MWD, 6-min walking distance; $\mathrm{EF}$, ejection fraction; VMI, ventricular mass index; RCWI, right cadiac work index; RHC, right heart catheterization; RVa, right ventricular diastole maximum area; RVd, right ventricular internal diameter; RVFAC, right ventricular fractional area change; RVSWI, right ventricular stroke work index; RVWT, right ventricular free wall thickness; SV, stroke volume; Tei, Tie index; $\mathrm{P}<0.05$.

Among these noninvasive measurements, CMRI is the optimal method to assess RV function, and then is the MDCT and the last is CUS.

\section{Funding}

the National 12th Five-Year Researching Foundation (2011BAI11B17).

\section{Authors' details}

${ }^{1}$ Department of Radiology, Beijing Chao-Yang Hospital, Capital Medical University, Beijing, China. ${ }^{2}$ Respiratory Diseases Researching Center of Capital Medical University, Beijing, China. ${ }^{3}$ Siemens Ltd., China, Beijing, China.

Published: 3 February 2015

doi:10.1186/1532-429X-17-S1-P185

Cite this article as: Guo et al:: Assessing right ventricular function in patients with pulmonary hypertension based on noninvasive measurements: correlation between cardiac MRI, ultrasonic cardiogram, multidetector CT and right heart catheterization. Journal of

Cardiovascular Magnetic Resonance 2015 17(Suppl 1):P185.
Submit your next manuscript to BioMed Central and take full advantage of:

- Convenient online submission

- Thorough peer review

- No space constraints or color figure charges

- Immediate publication on acceptance

- Inclusion in PubMed, CAS, Scopus and Google Scholar

- Research which is freely available for redistribution

Submit your manuscript at www.biomedcentral.com/submit 